\title{
A possible regulatory link between Twist 1 and PPARY gene regulation in 3T3-L1 adipocytes
}

\author{
Rui Ren', Zhufeng Chen', Xia Zhao', Tao Sun', Yuchao Zhang ${ }^{2}$, Jie Chen', Sumei Lu ${ }^{1 *+}$ and Wanshan Ma ${ }^{1+}$
}

\begin{abstract}
Background: Peroxisome proliferator-activated receptor $\gamma$ (PPARY) is a critical gene that regulates the function of adipocytes. Therefore, studies on the molecular regulation mechanism of PPARY are important to understand the function of adipose tissue. Twist 1 is another important functional gene in adipose tissue, and hundreds of genes are regulated by Twist 1. The aim of this study was to investigate the regulation of Twist 1 and PPARy expression in 3T3-L1 mature adipocytes.

Methods: We induced differentiation in 3T3-L1 preadipocytes and examined alterations in Twist 1 and PPARY expression. We used the PPARy agonist pioglitazone and the PPARy antagonist T0070907 to investigate the effect of PPARY on Twist 1 expression. In addition, we utilized retroviral interference and overexpression of Twist 1 to determine the effects of Twist 1 on PPARy expression.

Results: The expression levels of Twist 1 and PPARY were induced during differentiation in 3T3-L1 adipocytes. Application of either a PPARY agonist (pioglitazone) or antagonist (T0070907) influenced Twist 1 expression, with

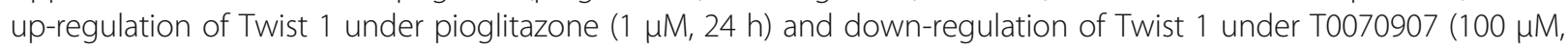
$24 \mathrm{~h}$ ) exposure. Furthermore, the retroviral interference of Twist 1 decreased the protein and mRNA expression of PPARY, while Twist 1 overexpression had the opposite effect.

Conclusions: There was a possible regulatory link between Twist 1 and PPARY in 3T3-L1 mature adipocytes. This regulatory link enhanced the regulation of PPARY and may be a functional mechanism of Twist 1 regulation of adipocyte physiology and pathology.
\end{abstract}

Keywords: Twist 1, PPAR Y, Adipose tissue, Gene regulation

\section{Background}

Adipose tissue is one of the most important functional organs in the human body and plays a critical role in human health. Adipose dysfunction is closely related to the occurrence and development of several diseases, including obesity, diabetes, insulin resistance and inflammation [1-3]. A number of genes are involved in the regulation of adipose function; however, the complex relationship between these genes has not been fully elucidated $[4,5]$. Therefore, it is important to explore the regulation and function of key genes involved in adipose function.

\footnotetext{
* Correspondence: Ismqianyi@126.com

${ }^{\dagger}$ Equal contributors

'Department of Laboratory Medicine, Shandong Provincial Qianfoshan Hospital,

Shandong University, Jinan, Shandong 250014, People's Republic of China

Full list of author information is available at the end of the article
}

Peroxisome proliferator-activated receptor $\gamma($ PPAR $\gamma)$ is a critical gene in the regulation of adipose function. Studies have shown that PPAR $\gamma$ is involved in several physiological functions of adipose, including adipocyte differentiation [6-8], adipokine secretion $[9,10]$, adipose tissue inflammation [11] and insulin sensitivity [12]. Therefore, investigating the regulation of PPAR $\gamma$ is important to further understand adipose function. Twist 1 is a well-conserved transcription factor that belongs to the basic helix-loop-helix (bHLH) family. Twist 1 has been shown to be important for the formation of adipose tissue, and recently, several studies have investigated the role of Twist 1 in adipose dysfunction. Twist 1 expression in subcutaneous adipose tissue and visceral adipose tissue is related to the pathogenesis of several metabolic 
syndromes, including obesity, inflammation and insulin resistance [13-15]. Therefore, PPAR $\gamma$ and Twist 1 are two genes with multiple similar functional roles in the regulation of adipose tissue.

However, it is unclear whether there is an interaction between Twist 1 and PPAR $\gamma$ in the functional regulation of adipose tissue. Recent studies have found that the function of Twist 1 in adipose tissue is closely related to PPAR family members. Twist 1 has been shown to be a PPAR delta-inducible, negative-feedback regulator of PGC-1 in brown fat metabolism, which has important implications for understanding metabolic control and metabolic diseases [16]. PPAR $\gamma$ has been shown to control the Twist 1-SMRT-GPS2 cascade, which is a regulatory transcriptional network involved in several functional processes in human adipocytes [17]. Previous studies from our laboratory also found evidence of Twist 1 and PPAR $\gamma$ regulation in 3T3-L1 preadipocytes. We showed that the retroviral interference of Twist 1 expression in 3T3-L1 preadipocytes enhances PPAR $\gamma$ expression after 4 days of hormone-induced differentiation [18]. These results indicate that the expression of Twist 1 and PPAR $\gamma$ are regulated by a complex interaction in adipose tissue. However, further studies are necessary to confirm the precise mechanism of this interaction.

The aim of this study was to explore whether changes in Twist 1 level influence PPARY expression. We used retroviral interference and Twist 1 overexpression to determine the role of Twist 1 on the regulation of PPARy. Also, we used a PPARy inhibitor and agonist to investigate the effect of PPAR $\gamma$ on Twist 1 expression. We verified that PPAR $\gamma$ regulates Twist 1 expression. In addition, we showed that Twist 1 could also influence PPARy expression positively. To our knowledge, the effect of Twist 1 on PPARY expression is a novel finding. We have enriched the knowledge of the regulation mechanism of PPAR $\gamma$, and these findings may explain the role of Twist 1 in adipocyte physiology and pathology.

\section{Methods \\ Reagents}

The 3T3-L1 mouse embryo fibroblasts and Dulbecco's modified Eagle medium (DMEM) were obtained from ATCC (Rockefeller, Maryland, USA). Bovine serum and fetal bovine serum (FBS) were purchased from GIBCO (Invitrogen, CA, USA). The primary antibodies (antiPPAR $\gamma$ and anti-Twist 1) were obtained from Abcam. The HRP-conjugated secondary antibody and the anti- $\beta$ actin primary antibody were purchased from ZSGB-BIO. The lentiviral vectors pGLV3/H1/GFP + Puro (LV3), LV4EF1a-GFP/Puro (LV5), recombinant vectors LV3/Twist 1 shRNA and LV5/Twist 1 complementary DNA (cDNA) were all acquired from GenePharma (Shanghai, China). NE-PER ${ }^{\text {Tw }}$ Nuclear and Cytoplasmic Extraction Kit was purchased from Pierce (Thermo Scientific). SYBR Green Real-time PCR Master Mix (QPK-201 T) was purchased from TOYOBO. T0070907 and pioglitazone were both from Cayman Chemical (MI, USA). Insulin, isobutylmethylxanthine, cycloheximide (CHX), leupeptin, pepstatin A (L/P), proteasomes inhibitors (MG132) and the other reagents in this study were obtained from Sigma (Merck, German).

\section{Induction of differentiation and Oil Red $O$ staining in 3T3-L1 preadipocytes}

The 3T3-L1 preadipocytes were maintained in DMEM supplemented with $10 \%$ bovine serum, $100 \mathrm{U} / \mathrm{ml}$ of penicillin and $100 \mathrm{mg}$ of streptomycin at $37^{\circ} \mathrm{C}$ in a humidified atmosphere composed of $95 \%$ air and $5 \% \mathrm{CO}_{2}$. The induction of differentiation and the Oil Red $\mathrm{O}$ staining were conducted according to previously described methods [17].

\section{Twist 1 shRNA and Twist 1 cDNA lentiviral vector construction and packaging}

The Twist 1 shRNA and Twist 1 cDNA lentiviral vectors were constructed and packaged by GenePharma. Twist 1 shRNA was generated based on previously described methods [17]. The recombinant vector, LV3/ Twist 1 shRNA, and the negative control vector, LV3/ $\mathrm{NC}$, were identified using sequencing. For packaging, a lentiviral vector titer of $1 \times 10^{9} \mathrm{TU} / \mathrm{mL}$ was used in the infection experiments.

The full Twist $1 \mathrm{cDNA}$ sequence was synthesized using chemical methods. The Kozak protection sequence (GCCACC) and a NotI site (GCGGCCGC) were added to the $5^{\prime}$-end of the Twist 1 cDNA. A BamHI site (GGATCC) was added to the 3 '-end of the Twist 1 cDNA. This complex was then inserted into the expression vector LV4-EF1a-GFP/Puro (LV5), which also served as a negative control in this study. A lentiviral vector titer of $1 \times 10^{9}$ $\mathrm{TU} / \mathrm{ml}$ of LV5/Twist 1 cDNA was used in the infection experiments.

\section{Lentiviral vector infection with LV3/Twist1 shRNA and LV5/Twist 1 cDNA}

Mature adipocytes were infected with the lentiviral vectors (LV3/Twist1 shRNA, LV3/NC, LV5/Twist 1 cDNA and LV5/NC) $6 \mathrm{~d}$ after the induction of differentiation. The proportion of the lentiviral vector and high-glucose DMEM supplemented with 10 \% FBS was 1:20. The cells were incubation for $8-12 \mathrm{~h}$, and then, the infection medium was replaced with culture medium containing $10 \% \mathrm{FBS}$ and incubated for $48 \mathrm{~h}$.

\section{Extraction of cytoplasmic and nuclear proteins}

Cytoplasmic and nuclear proteins were extracted using a NE-PER ${ }^{\text {Tw }}$ Nuclear and Cytoplasmic Extraction Kit (Prod\# 78833) according to the manufacturer's instructions. Briefly, 
the cells were washed three times, harvested with $1 \mathrm{ml}$ of PBS and centrifuged at 500xg for $5 \mathrm{~min}$ in microcentrifuge tubes. Ice-cold CER I buffer was added to homogenize the cells, and the homogenate was vigorously vortexed for $15 \mathrm{~s}$ and incubated for $10 \mathrm{~min}$ on ice. Next, ice-cold CER II buffer was added, and the mixture was vortexed for $5 \mathrm{~s}$ and incubated for $1 \mathrm{~min}$. The mixture was centrifuged for $5 \mathrm{~min}$ at 16,000xg and the supernatant solution was transferred to a fresh microcentrifuge tube as the cytoplasmic protein fraction. The remaining pellet was suspended in ice-cold NER buffer and then vortexed for 40 min with 15$\mathrm{s}$ breaks every $10 \mathrm{~min}$. Finally, the samples were centrifuged at $16,000 \mathrm{xg}$ for $15 \mathrm{~min}$ to obtain the supernatant as the nuclear fraction.

\section{RNA extraction and real-time PCR detection}

Total RNA was extracted using TRIzol (Invitrogen) according to conventional experimental procedure, and cDNA was synthesized based on standard protocols of RevertAid First Strand cDNA Synthesis kit (Thermo Scientific). Real-time PCR detection was conducted using the SYBR Green Real-Time PCR Master Mix Kit according to the manufacturer's instructions. The PCR primers were designed using Primer 5.0 software, and the primer sequences has been shown in Table 1. The relative quantification of the target sequence was determined as the fold changes compared with GAPDH mRNA using the 2- $\Delta \Delta \mathrm{Ct}$ method. All reactions were conducted in triplicate.

\section{Western blot analysis}

Western blotting was conducted according to previously described methods [18]. The membranes were incubated overnight at $4{ }^{\circ} \mathrm{C}$ with primary antibodies for antiPPAR $\gamma$ (1:1,000), anti-Twist1 (1:50) and anti- $\beta$-actin (1:2500). The target molecular weights for Twist 1 , PPAR $\gamma$ and $\beta$-actin were $21 \mathrm{kDa}, 58 \mathrm{kDa}$ and $43 \mathrm{kDa}$, respectively. The relative OD ratio was calculated using ImageJ software and normalized to $\beta$-actin. Data from three replicate experiments were analyzed.

\section{Statistical analyses}

For Oil Red O staining, the data are presented as the OD values. ImageJ software was used to quantify the results from the western blotting experiment. The relative gray value of each band was calculated based on results of three replicates. All data are shown as the mean \pm SD, and the data were analyzed using SPSS software package, version 17.0. $\mathrm{T}$ tests were used for comparisons between two groups. A one-way analysis of variance (ANOVA) was performed for comparisons between more than two groups, and Dixon's $q$ tests were used for further pairwise comparisons. $P<0.05$ was considered significant.

\section{Results}

Twist 1 and PPARy expression is induced during 3T3-L1 differentiation

Differentiation was induced in 3T3-L1 preadipocytes according to previously described methods, and obvious morphological changes were observed during differentiation. As shown in Fig. 1a, the confluent 3T3-L1 preadipocytes exhibited a long shuttle shape without lipid droplets. During differentiation, a portion of the adipocytes began to accumulate lipids, which spread until most of the adipocytes were full of lipid droplets at day 8-12 after the initiation of induction. Lipid accumulation was quantified using Oil Red O staining (Fig. 1b), and significant differences in lipid content were observed $4 \mathrm{~d}$ after differentiation $(* P<0.05)$.

The mRNA and protein expression of Twist 1 and PPAR $\gamma$ in 3T3-L1 adipocytes were confirmed using real time PCR and western blotting, respectively. Figure 1c shows that there were significant increases in the mRNA levels of Twist 1 and PPAR $\gamma$ in the mature 3T3-L1 adipocytes $\left({ }^{*} P<0.05\right)$. In addition, Twist 1 and PPAR $\gamma$ protein expression levels were significantly upregulated in mature 3T3-L1 adipocytes compared with the 3T3-L1 preadipocytes (Fig. 1d, e, " $P<0.05$ ).

\section{Application of pioglitazone and T0070907 alters Twist 1 expression levels}

To determine how PPARy regulates Twist 1 expression, we added pioglitazone ( $1 \mu \mathrm{M}, 24 \mathrm{~h})$, a classic PPAR $\gamma$ agonist, and T0070907 $(100 \mu \mathrm{M}, 24 \mathrm{~h})$, a common PPAR $\gamma$ inhibitor, into the culture medium of 3T3-L1 mature adipocytes and determined the protein expression of Twist 1. Taken the relative Twist 1 expression in both control group as $100 \%$, the expression level of Twist 1 in the

Table 1 Sequence information on the primers used for RT-PCR

\begin{tabular}{lllll}
\hline Genes & Sequences & Product size (bp) & Annealing temperature $\left({ }^{\circ} \mathrm{C}\right)$ & Gene Bank \\
\hline Mouse Twist 1 & 5'-CATGGCTAACGTGCGGGA-3' & 124 & 60 & NM_011658.2 \\
Mouse PPARY & 5'-CGCCAGTTTGAGGGTCTGAA-3' & & & NM_001127330.2 \\
& 5'-GCTGACCCAATGGTTGCTGA-3' & 181 & 60.5 & \\
GAPDH & 5'-CTTTATCCCCACAGACTCGGC-3' & & 59 & NM_001289726.1 \\
& 5'-TGGCCTTCCGTGTTCCTAC-3' & 178 & & \\
\hline
\end{tabular}



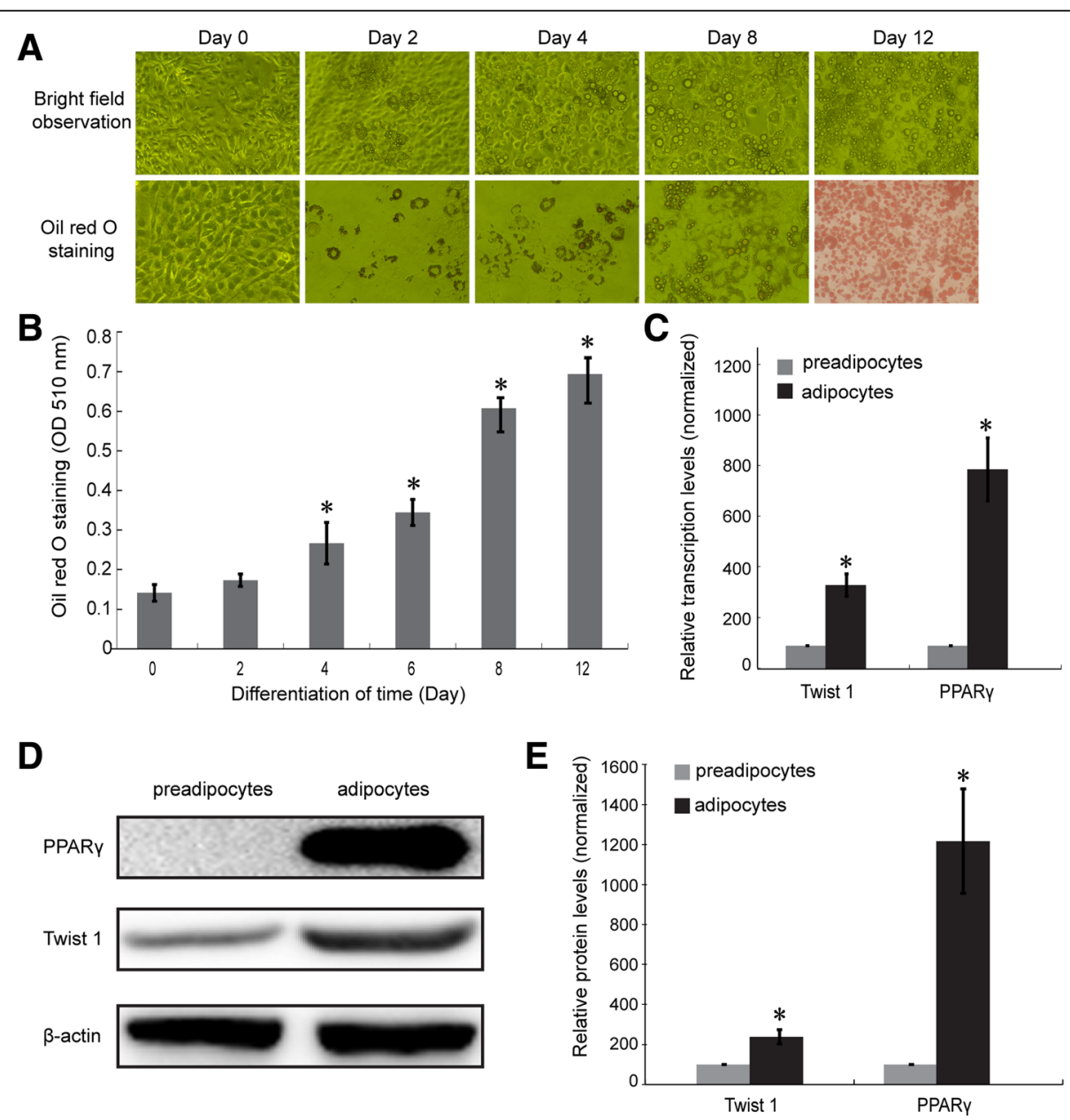

Fig. 1 The induction of differentiation and the confirmation of Twist 1 and PPARY expression in 3T3-L1 adipocytes. a The morphological changes observed in 3T3-L1 preadipocytes during differentiation under bright field and Oil Red O staining. b Quantification of lipids based on Oil Red O staining. c The transcription levels of Twist 1 and PPARy were both elevated in adipocytes. $\mathbf{d}$ Twist 1 and PPARy protein expression levels were upregulated during adipogenesis. e Quantification of the protein expression using ImageJ software

pioglitazone $(1 \mu \mathrm{M}, 24 \mathrm{~h})$ exposure group was significantly upregulated $(164.3 \pm 20.51 \%, P<0.05)$ (Fig. 2a, b), while that in the T0070907 $(100 \mu \mathrm{M}, 24 \mathrm{~h})$ treatment group was $(30.51 \pm 4.62) \%$, significantly down-regulated $(P<0.05)$ (Fig. 2c, d).

\section{Retroviral interference or overexpression of Twist 1 regulates PPARY expression by targeting protein synthesis}

LV3/Twist1 shRNA (LV3/Twist 1- group) reduced Twist 1 expression to $(44.80 \pm 7.48) \%$ compared with the control and LV3/NC group $(P<0.05)$, which suggest that retroviral interference was effective. Taken the relative PPAR $y$ expression in control group as $100 \%$, the expression level of PPAR $\gamma$ in the LV3/Twist 1- group was $(57.75 \pm 5.66) \%$, significantly downregulated $(P<0.05)$ (Fig. 3a, b). While as shown in Fig. 3c, d, Twist 1 overexpression by the lentivirus vector LV4-EF1a-GFP/Puro (LV5) significantly increased Twist 1 expression to
$(158.12 \pm 7.23) \%$ in the LV5/Twist $1+$ group $(P<0.05)$. Correspondingly, PPAR $\gamma$ expression was increased in the LV5/Twist $1+$ group to $(141.16 \pm 14.55 \%, P<0.05)$.

Increased protein synthesis or decreased protein degradation might be responsible for the upregulation of PPAR $\gamma$ expression observed in our experiments. To confirm which pathway is involved, we incubated the 3T3-L1 adipocytes with either a protein synthesis inhibitor ( $\mathrm{CHX})$ or protease inhibitor $(\mathrm{L} / \mathrm{P})$ and proteasome inhibitor (MG132) $1 \mathrm{~h}$ before treatment with the LV5/Twist 1+ lentivirus vector. As shown in Fig. 3e, f, $\mathrm{CHX}$ abolished the LV5/Twist $1+$-induced increase in PPAR $\gamma$ expression $\left({ }^{*} P<0.05\right)$, while $\mathrm{L} / \mathrm{P}+\mathrm{MG} 132$ application had no effect on the levels of PPAR $\gamma$.

\section{PPAR transcription was confirmed under retroviral interference or overexpression of Twist 1}

Given that Twist 1 regulates PPAR $\gamma$ expression by influencing protein synthesis, we next investigated transcriptional 

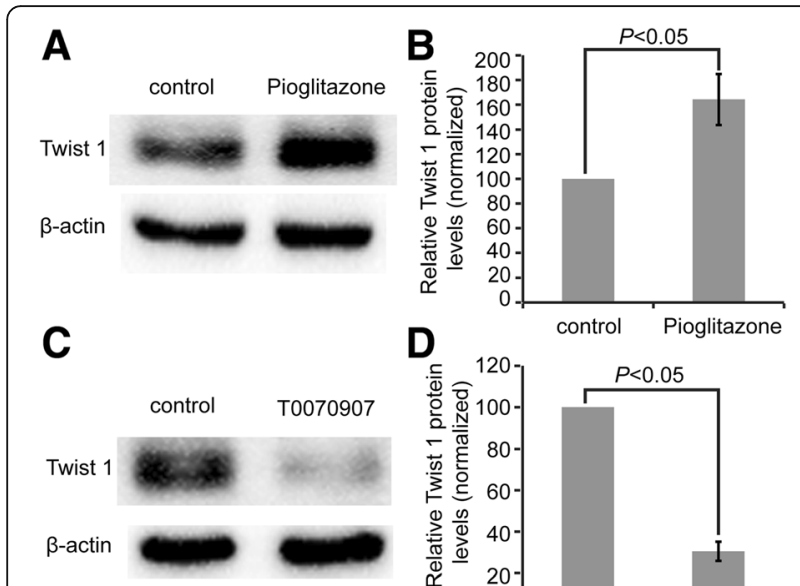

D

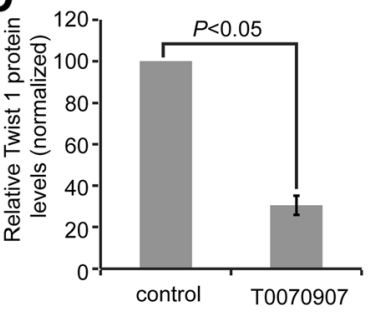

Fig. 2 T0070907 and pioglitazone treatment changed Twist 1 expression. a The effect of pioglitazone $(1 \mu \mathrm{M}, 24 \mathrm{~h})$ application on Twist 1 expression assessed by western blotting. $\mathbf{b}$ The relative quantification of Twist 1 expression after pioglitazone treatment. c T0070907 treatment (100 $\mu \mathrm{M}, 24 \mathrm{~h})$ decreased Twist 1 expression, as detected by western blotting. $\mathbf{d}$ Quantification of the Twist 1 protein expression under T0070907 exposure using ImageJ software

regulation of PPAR $\gamma$ to confirm this finding. As shown in Fig. 4a, retroviral interference of Twist 1 (LV3/Twist 1-) significantly decreased the mRNA expression of PPAR $\gamma$ transcription $(62.2 \pm 8.24 \%)$ compared with the control group (set at $100 \%$ ). Twist 1 overexpression significantly increased PPAR $\gamma$ mRNA expression (179.53 \pm 19.54 \%; ${ }^{*} P<0.05$ ) (Fig. 4b).

\section{Discussion}

In the present study, we investigated the probable role of Twist 1 in the regulation of PPARy expression. We found PPAR $\gamma$ and Twist 1 were both upregulated during adipogenesis. Application of pioglitazone increased Twist 1 expression and T0070907 exposure decreased Twist 1 expression level. Similarly, retroviral interference or overexpression of Twist 1 positively regulated PPAR $\gamma$ levels by influencing protein synthesis. To our knowledge, this is the first study to report that Twist 1 regulates PPAR $\gamma$ expression; however, PPAR $\gamma$ control of Twist 1 expression has been previously reported [17].

We have previously successfully induced mature and stable differentiation of adipocytes from preadipocytes [19]. In this study, we observed morphological changes that indicate adipogenesis (including the appearance of lipids) from day 4 after the induction of differentiation. PPAR $\gamma$ regulates adipocyte differentiation, and PPAR $\gamma$ upregulation is necessary and sufficient for adipocyte formation [20]. We detected PPARY upregulation in adipocytes during differentiation, which is consistent with previous reports $[17,18,21]$. In addition, in this study, we showed

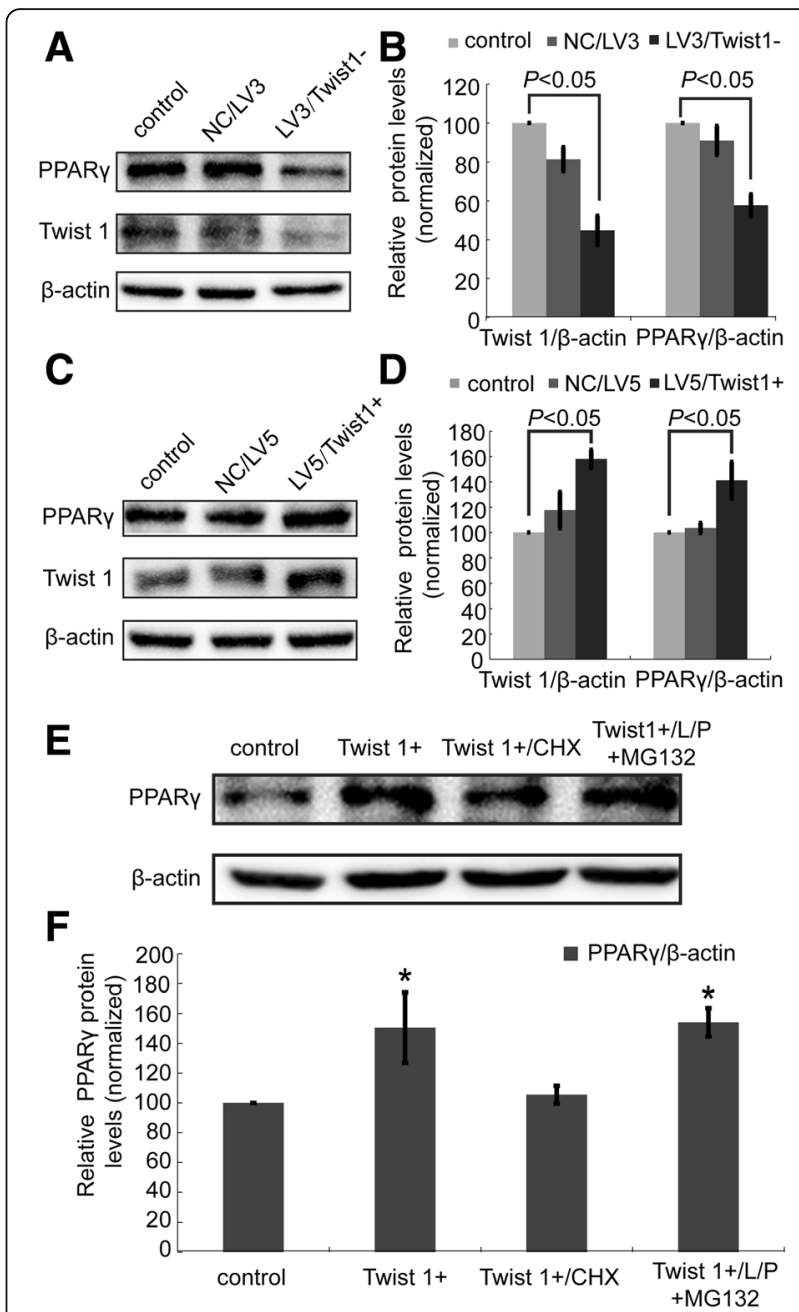

Fig. 3 Retroviral interference or overexpression of Twist 1 positively regulated PPARY expression by influencing PPARY protein synthesis. a Twist 1 and PPARy expression after shRNA targeted Twist 1 interference. $\mathbf{b}$ Quantification of the protein expression in (a) using ImageJ software. c Twist 1 and PPARy expression after the overexpression of Twist 1 cDNA. $\mathbf{d}$ Quantification of the protein expression in (c) using ImageJ software. e Alterations in PPARY protein synthesis and protein degradation after treatment with either $C H X$ or L/P + MG132. f Quantification of the protein expression in (e) using ImageJ software

that Twist 1 was upregulated during adipogenesis, which has been confirmed in our previous studies [18]. Overexpression of Twist 1 has also been shown to enhance the capacity of purified human bone marrow-derived mesenchymal stromal/stem cells to undergo adipogenesis [22]. Similarly, our results suggest that Twist 1 has a critical function in adipocyte differentiation. These preliminary results suggested that maybe there are some crossfunctions for Twist 1 and PPAR $\gamma$ in adipocytes, indicated the possibility of probable regulation between Twist 1 and PPAR $\gamma$. Further studies about the regulatory link between Twist 1 and PPAR $\gamma$ were necessary and meaningful. 


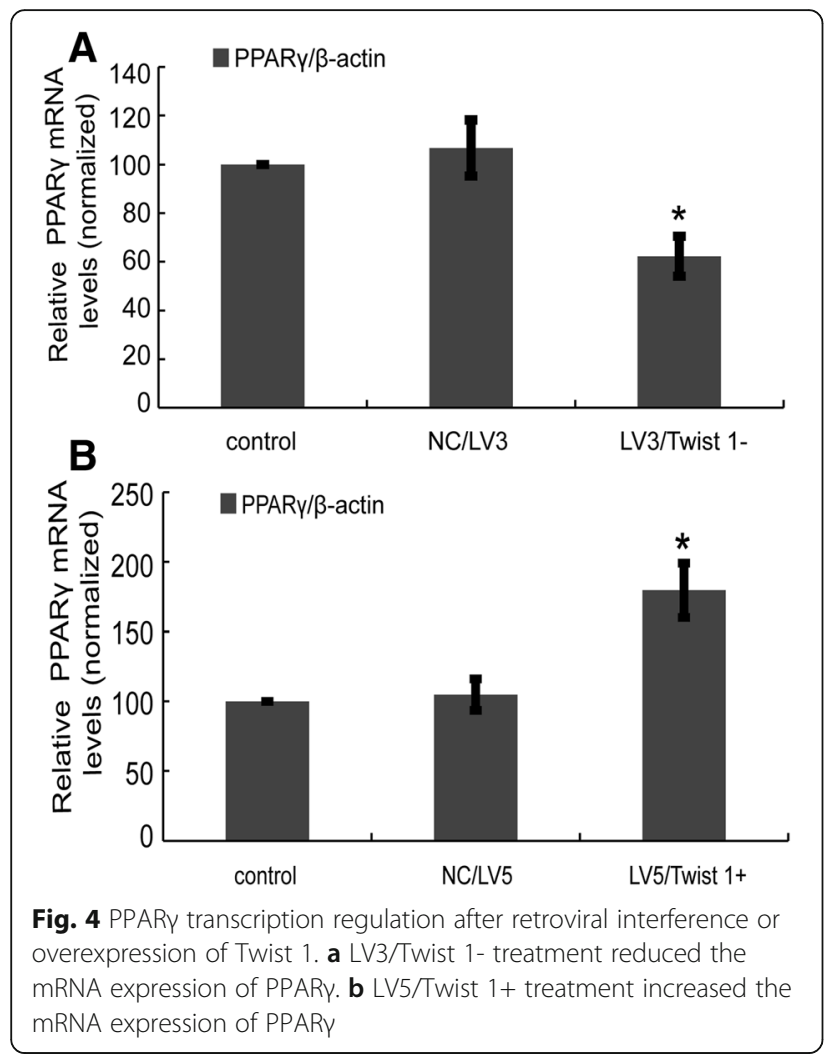

The PPAR $\gamma$ antagonist T0070907 has been widely used to inhibit PPARY activity [23-25]. In this study, we proved that Twist 1 expression of 3T3-L1 adipocytes was downregulated under T0070907, which indicates that PPAR $\gamma$ downregulation inhibits Twist 1 expression. Several studies have also used the PPAR $\gamma$ agonist pioglitazone to investigate the function of PPARY [26]. The positive regulation between PPAR $\gamma$ and Twist 1 expression levels was also observed after pioglitazone treatment in the current study, with obvious up-regulation of Twist 1 in pioglitazone exposure group. This finding suggests that PPAR $\gamma$ positively controls Twist 1 expression. Similarly, studies in diabetic obese patients found that pioglitazone treatment increased Twist 1 expression in adipose tissue and showed that PPAR $\gamma$ controlled Twist 1 expression through the Twist 1-SMRT-GPS2 cascade, which is consistent with our findings [17].

We used retroviral interference or overexpression of Twist 1 to control Twist 1 expression. The results showed that Twist 1 downregulation significantly reduced PPAR $\gamma$ expression. Conversely, Twist 1 overexpression significantly increased PPAR $\gamma$ expression. These results indicate that alterations in Twist 1 expression positively regulate PPAR $\gamma$, which has not been shown previously. As an important member of the bHLH family of transcription factors, hundreds of genes are regulated by Twist 1, including miRNAs $[27,28]$ and genes related to the epithelial-to-mesenchymal transition (EMT) in carcinomas $[29,30]$. In addition, Hes4 is a novel bHLH transcription factor that has been shown to be regulated by Twist 1 and antagonize the function of Twist 1 in regulating lineage commitment of bone marrow stromal/stem cells [31]. In this study, we suggest that PPARY is also under the regulation of Twist 1.

Two opposite processes may be responsible for the observed changes in protein expression: protein synthesis and protein degradation. An increase in protein expression may result from increased protein synthesis or decreased protein degradation. Therefore, we used $\mathrm{CHX}$ and L/P + MG132 to determine whether the regulation of PPAR $\gamma$ by Twist 1 was a result of changes in protein synthesis or degradation. Our results confirmed that the retroviral interference or overexpression of Twist 1 altered the protein synthesis, and not the protein degradation, of PPAR $\gamma$, which was further confirmed by assessing the transcriptional regulation of PPAR $\gamma$.

It is worth noting that, there seems to be some discrepancy between the results of our previous report [18] and the current results for the regulation relationship between Twist 1 and PPAR $\gamma$ expression. In our previous work, Twist 1 lentivirus interference increased PPAR gamma expression in the day $4^{\text {th }}$ differentiation induction cells, while a downregulation of PPAR $\gamma$ was reported under Twist 1 lentivirus interference in the current study. In fact, the research material is quite different between the present study and the previous work [18], which should be responsible for the main inconsistence. In our previous work, we infected Twist 1 interference lentivirus to the 3T3-L1 preadipocytes, and found an increase in PPARy expression at day $4^{\text {th }}$ differentiation induction under interference of Twist 1. However, in the present study, mature adipocytes were our research objects, and Twist 1 interference lentivirus was infected to 3T3-L1 mature adipocytes after 12-14 days differentiation induction. As we know, adipogenesis process was a complex process regulated by multi-genes, adipocytes located in the day $4^{\text {th }}$ differentiation induction was not yet stable, and was a form of transitional cell phase, which means lots of instability and complexity. It can't be excluded that the increase of PPAR $\gamma$ expression at day $4^{\text {th }}$ differentiation induction may not be the direct effect of Twist 1. But mature adipocytes experienced after 12-14 days differentiation induction were stable, without these above concerns. Further, in the present study, we introduced Twist 1 overexpression and PPAR gamma inhibitor to explore the full regulation relationship between Twist 1 and PPAR gamma, which can reflect the relationship of both genes from different levels.

In summary, in this study, we reported a possible regulatory link between Twist 1 and PPAR $\gamma$, especially the probable role of Twist 1 in regulating PPARy expression. PPARY has been shown to control the Twist 1- 
SMRT-GPS2 cascade in human adipocytes [17]; however, our results suggest that an interaction between Twist 1 and PPAR $\gamma$ probably exists in 3T3-L1 adipocytes. This regulatory link significantly enriched the gene regulation mechanism of PPAR $\gamma$ and may explain the role of Twist 1 in adipocyte physiology and pathology.

Although not used in this study, a ChIP assay would definitively show the role of Twist 1 in regulating PPAR $\gamma$. However, our results provide evidence of a regulatory link between Twist 1 and PPAR $\gamma$, which indicate a possible mechanism of Twist 1 in adipocytes function. Further studies are necessary to fully elucidate the relationship between PPAR $\gamma$ and Twist 1 in adipocyte function.

\section{Conclusion}

In this study, a possible regulatory link between Twist 1 and PPAR $\gamma$ in 3T3-L1 mature adipocytes was reported. This regulatory link enriched the regulation mechanism of PPARY and may also be critical mechanisms involved in Twist 1 functions in adipocyte physiology and pathology. This study provides theoretical basis for further studies about the role and mechanism of PPAR $\gamma$ and Twist 1 in adipocytes.

\section{Acknowledgements}

We thank American Journal Experts (AJE) for the English language service about this manuscript.

\section{Funding}

This work was supported by the National Natural Science Foundation (No. 81400843 No. 81541021, and No. 81501226), and Shandong Provincial Natural Science Foundation (No. ZR2014HP033).

\section{Availability of data and materials}

The datasets analyzed during the current study are available from the corresponding author on reasonable request.

\section{Authors' contributions}

WSM and SML conceived and designed the experiments; WSM, SML, RR, ZFC, and ZX performed the experiments. RR, ZX, TS, YCZ and JC analyzed the data. WSM and SML wrote the paper. All authors read and approved the final version of the manuscript.

\section{Competing interests}

The authors declare that they have no competing interests.

\section{Consent for publication}

Not applicable.

\section{Author details \\ 'Department of Laboratory Medicine, Shandong Provincial Qianfoshan Hospital, Shandong University, Jinan, Shandong 250014, People's Republic of China. ²Department of Endocrinology, Qingdao Municipal Hospital, Qingdao 266071, People's Republic of China.}

Received: 5 May 2016 Accepted: 2 November 2016 Published online: 08 November 2016

\section{References}

1. Bremer AA, Jialal I. Adipose tissue dysfunction in nascent metabolic syndrome. J Obes. 2013;2013:393192.

2. Zhang H, Sairam MR. Sex hormone imbalances and adipose tissue dysfunction impacting on metabolic syndrome; a paradigm for the discovery of novel adipokines. Horm Mol Biol Clin Investig. 2014;17(2):89-97.
3. Spritzer PM, Lecke SB, Satler F, Morsch DM. Adipose tissue dysfunction, adipokines, and low-grade chronic inflammation in polycystic ovarysyndrome. Reproduction. 2015;149(5):R219-27.

4. Stanford KI, Middelbeek RJ, Goodyear LJ. Exercise effects on White adipose tissue: Beiging and metabolic adaptations. Diabetes. 2015;64(7):2361-8.

5. Shostak A, Husse J, Oster $\mathrm{H}$. Circadian regulation of adipose function. Adipocyte. 2013;2(4):201-6.

6. Farmer SR. Transcriptional control of adipocyte formation. Cell Metab. 2006; 4(4):263-73.

7. Siersbaek R, Nielsen R, Mandrup S. PPARgamma in adipocyte differentiation and metabolism-novel insights from genome-wide studies. FEBS Lett. 2010; 584(15):3242-9.

8. Moseti D, Regassa A, Kim WK. Molecular regulation of adipogenesis and potential anti-adipogenic bioactive molecules. Int J Mol Sci. 2016;17(1):124

9. Parimisetty A, Dorsemans AC, Awada R, Ravanan P, Diotel N, Lefebvre d'Hellencourt C. Secret talk between adipose tissue and central nervous system via secreted factors-an emerging frontier in the neurodegenerative research. J Neuroinflammation. 2016;13(1):67.

10. Jin D, Sun J, Huang J, Yu X, Yu A, He Y, Li Q, Yang Z. Peroxisome proliferator-activated receptor $\gamma$ enhances adiponectin secretion via up-regulating DsbA-L expression. Mol Cell Endocrinol. 2015:411:97-104.

11. Polvani S, Tarocchi M, Tempesti S, Bencini L, Galli A. Peroxisome proliferator activated receptors at the crossroad of obesity, diabetes, and pancreatic cancer. World J Gastroenterol. 2016;22(8):2441-59.

12. Kennedy A, Martinez K, Chuang CC, LaPoint K, Mclntosh M. Saturated fatty acid-mediated inflammation and insulin resistance in adipose tissue: mechanisms of action and implications. J Nutr. 2009;139(1):1-4.

13. Pettersson AT, Laurencikiene J, Mejhert N, Näslund E, Bouloumié A, Dahlman I, Arner P, Rydén M. A possible inflammatory role of twist1 in human white adipocytes. Diabetes. 2010:59(3):564-71.

14. Dobrian AD. A tale with a twist: a developmental gene with potential relevance for metabolic dysfunction and inflammation in adipose tissue. Front Endocrinol. 2012;3:108.

15. Pettersson AT, Mejhert N, Jernås M, Carlsson LM, Dahlman I, Laurencikiene J, Arner P, Rydén M. Twist 1 in human white adipose tissue and obesity. J Clin Endocrinol Metab. 2011;96(1):133-41.

16. Pan D, Fujimoto M, Lopes A, Wang YX. Twist-1 is a PPARdelta-inducible, negative-feedback regulator of PGC-1alpha in brown fat metabolism. Cell. 2009;137(1):73-86

17. Toubal A, Clément K, Fan R, Ancel P, Pelloux V, Rouault C, Veyrie N, Hartemann A, Treuter E, Venteclef N. SMRT-GPS2 corepressor pathway dysregulation coincides with obesity-linked adipocyte inflammation. J Clin Invest. 2013;123(1):362-79.

18. Ma W, Lu S, Sun T, Wang X, Ma Y, Zhang X, Zhao R, Wang Y. Twist 1 regulates the expression of PPARY during hormone-induced 3T3-L1 preadipocyte differentiation: a possible role in obesity and associated diseases. Lipids Health Dis. 2014;13:132.

19. Lu S, Guan Q, Liu Y, Wang H, Xu W, Li X, Fu Y, Gao L, Zhao J, Wang X. Role of extrathyroidal TSHR expression in adipocyte differentiation and its association with obesity. Lipids Health Dis. 2012;11:17.

20. Rosen ED, Sarraf P, Troy AE, Bradwin G, Moore K, Milstone DS, Spiegelman $\mathrm{BM}$, Mortensen RM. PPAR gamma is required for the differentiation of adipose tissue in vivo and in vitro. Mol Cell. 1999;4(4):611-7.

21. Jun HJ, Gettys TW, Chang JS. Transcriptional activity of PGC-1a and NT-PGC$1 a$ is differentially regulated by twist- 1 in Brown fat metabolism. PPAR Res. 2012;2012:320454

22. Isenmann S, Arthur A, Zannettino AC, Turner JL, Shi S, Glackin CA, Gronthos S. TWIST family of basic helix-loop-helix transcription factors mediate human mesenchymal stem cell growth and commitment. Stem Cells. 2009; 27(10):2457-68.

23. Schaefer KL. PPARgamma inhibitors as novel Tubulin-Targeting agents. PPAR Res. 2008;2008:785405.

24. An Z, Muthusami S, Yu JR, Park WY. T0070907, a PPAR y inhibitor, induced G2/M arrest enhances the effect of radiation in human cervical cancer cells through mitotic catastrophe. Reprod Sci. 2014;21(11):1352-61.

25. Yang $K$, Lu W, Jiang Q, Yun X, Zhao $M$, Jiang $H$, Wang J. Peroxisome proliferator-activated receptor $\gamma$-mediated inhibition on hypoxia-triggered store-operated calcium entry. A caveolin-1-dependent mechanism. Am J Respir Cell Mol Biol. 2015;53(6):882-92. 
26. Zhao D, Zhu Z, Li D, Xu R, Wang T, Liu K. Pioglitazone suppresses CXCR7 expression to inhibit human macrophage chemotaxis through Peroxisome proliferator-activated receptor $\gamma$. Biochemistry. 2015;54(45):6806-14.

27. Sokol NS, Ambros V. Mesodermally expressed drosophila microRNA-1 is regulated by twist and is required in muscles during larvalgrowth. Genes Dev. 2005:19(19):2343-54.

28. Jensen ED, Gopalakrishnan R, Westendorf JJ. Regulation of gene expression in osteoblasts. Biofactors. 2010;36(1):25-32

29. Wushou A, Hou J, Zhao YJ, Shao ZM. Twist-1 up-regulation in carcinoma correlates to poor survival. Int J Mol Sci. 2014;15(12):21621-30.

30. Teng $Y, L i X$. The roles of HLH transcription factors in epithelial mesenchymal transition and multiple molecular mechanisms. Clin Exp Metastasis. 2014;31(3):367-77.

31. Cakouros D, Isenmann S, Hemming SE, Menicanin D, Camp E, Zannetinno AC, Gronthos S. Novel basic helix-loop-helix transcription factor hes4 antagonizes the function of twist-1 to regulate lineage commitment of bone marrow stromal/stem cells. Stem Cells Dev. 2015:24(11):1297-308.

Submit your next manuscript to BioMed Central and we will help you at every step:

- We accept pre-submission inquiries

- Our selector tool helps you to find the most relevant journal

- We provide round the clock customer support

- Convenient online submission

- Thorough peer review

- Inclusion in PubMed and all major indexing services

- Maximum visibility for your research

Submit your manuscript at www.biomedcentral.com/submit
Biomed Central 